Revista Científica de FAREM-Estelí

Medio ambiente. tecnología y desarrollo humano

Año 10 | Edición especial: artículos de revisión documental

ISSN: 2305-5790

https://rcientificaesteli.unan.edu.ni

DOI: https://doi.org/10.5377/farem.v0i0.11610

\section{La gestión empresarial en las micro, pequeñas, medianas empresas}

\section{Business management in micro, small and medium sized enterprises}

\section{Arlen Meryfel Picado Juárez}

Maestría en Gestión, sostenibilidad y calidad de las MIPYMES (UNAN-Managua), estudiante de doctorado en Gestión y Calidad de la Investigación Científica II Cohorte 2020-2023 (UNAN-Managua). Docente de la Universidad Nacional Autónoma de Nicaragua, FAREM-Estelí. https://orcid.org/0000-0002-8817-7841

arlenmery@yahoo.com

\section{Natalia Sergueyevna Golovina}

Tutora de tesis doctoral, PhD. en Ciencias Sociales, profesora Titular de UNANManagua, FAREM-Matagalpa.

https://orcid.org/0000-0001-9081-6748

natygolovina@gmail.com
RECIBIDO

26/03/2021

ACEPTADO

19/052021

\title{
RESUMEN
}

PALABRAS CLAVE

La gestión empresarial se sustenta en diversas teorías para su aplicación; de ahí, el propósito de este artículo, la compilación de los fundamentos teóricos y su aplicación en el área administrativa de las empresas. Se sustenta en postulados teóricos como la científica de Frederick Taylor y la clásica de Henry Fayol, entre otros. Estas teorías tienen relación con la gestión empresarial en las micro, pequeñas, medianas empresas (mipyme). Se manifiesta la relación de los enfoques teóricos con la gestión empresarial y su aplicación para el manejo y funcionamiento de cualquier empresa sin importar su tamaño o giro del negocio. La metodología utilizada consistió en la revisión bibliográfica de fuentes relacionadas con las teorías administrativas y gestión empresarial, en libros, artículos de revistas científicas y tesis doctorales, consultas en las bases de datos suscritas de la Universidad Nacional Autónoma de Nicaragua, Managua (UNAN-Managua), el repositorio Centroamericano del Consejo Nacional de Universidades (CNU) y Google académico. La puesta en práctica de la gestión empresarial permitirá a los empresarios visualizar la importancia que tiene la ejecución de la gestión administrativa para la mejora continua de los procesos, el logro de los objetivos, optimización de recursos para alcanzar el desarrollo, éxito y sostenibilidad empresarial. 


\section{ABSTRACT}

Business management is based on various theories for its application; the purpose of this article is the compilation of the theorical foundations and their application in the administrative area of companies. It is based on theoretical postulates such as the scientific theory of Frederick Taylor and the classical theory of Henry Fayol, among others. These theories are relation to business management in the micro, smalls and medium-sized enterprises (MSMEs). The relationship of the theoretical approaches with business management and their application to the management and operation of any company, regardless of its size or line of business, is shown. The methodology used consisted in the bibliographic review of the sources related with the administrative theories and business management, in books, articles of scientific journal articles and doctoral theses, consultations in the subscribed databases of the National Autonomous University of Nicaragua, Managua (UNAN-Managua), the Central American repository of the National Council of Universities (CNU) and Google Academic. The implementation of business management will allow entrepreneurs to visualize the importance of the execution of administrative management for the continuous improvement of processes, the achievement objectives, optimization of resources to achieve business development, success and sustainability.

\section{KEYWORDS}

Theories; companies; business management; continuous improvement. 


\section{INTRODUCCIÓN}

El ambiente empresarial ha cambiado en el contexto nacional e internacional de forma dinámica y competitiva. Las exigencias del cliente cambian, ya no es fácil venderle un producto o servicio, por ello los empresarios deben desarrollar estrategias para competir en el sector y con el paso del tiempo alcanzar la sostenibilidad en el mercado. Hablar de sostenibilidad no solo es permanecer en el mercado, sino emplear los recursos con los que cuenta la empresa a través de una buena y adecuada gestión empresarial.

En este contexto complejo y dinámico es indispensable que el empresario cuente con una visión de futuro, así podrá afrontar las distintas circunstancias que modifican el entorno y que influyen en la gestión empresarial del negocio. Los emprendimientos pueden estar siendo afectados por diversos factores internos y externos y atravesando problemas administrativos para mantenerse en el sector que atienden y teniendo dificultades para el logro de los objetivos a corto, mediano y largo plazo.

El propósito de la presente investigación documental, es la compilación de los fundamentos teóricos del proceso de gestión empresarial para proporcionar aportes a los empresarios en el área administrativa, que les permita disponer de conocimientos fundamentales para que puedan realizar una adecuada gestión empresarial, para el cumplimiento de los objetivos, la eficacia y eficacia de los procesos y los recursos de sus negocios.

Galarza, Mora y Zambrano (2020), "La gestión administrativa tiene un carácter sistémico, al ser portadora de acciones coherentemente orientadas al logro de los objetivos a través del cumplimiento de las funciones clásicas de la gestión en el proceso administrativo: planear, organizar, dirigir y controlar" (pág. 3) .

Es importante destacar que el conocimiento aportado en esta revisión documental permite contextualizarse con las empresas emprendedoras nicaragüenses, por el ambiente competitivo en el que se encuentran, luchando día a día por su estabilidad con empresas ya establecidas, las que cuentan con estructura formal, con muchos años de experiencia en el sector, también con personal capacitado, con capacidad instalada en producción y tecnología, esta es la competencia que acaparan el mercado de forma ágil y dinámica, las que son gestionadas con asertividad y conocimiento en lo administrativo, contable y financiero, elementos indispensables en todo negocio para alcanzar la rentabilidad y sostenibilidad en el sector.

Por lo anteriormente expuesto, se encuentra la limitante de que no existen investigaciones realizadas en el país con relación al tema presentado, que 
sirvan como referente para conocer a profundidad las diversas problemáticas que han venido enfrentando las empresas en el país.

La estructura del contenido inicia con los fundamentos teóricos de la administración, los que sustentan a la gestión administrativa, seguido de las áreas de la gestión empresarial y por último las conclusiones de artículo.

\section{MATERIALES Y MÉTODOS}

La metodología utilizada para elaborar este artículo es de revisión documental, la cual inicia con la investigación bibliográfica de fuentes relacionadas con las teorías administrativas y gestión empresarial. Para ello se consultaron las bases de datos suscritas de la UNAN-Managua, CNU y Google académico. Las fuentes consultadas fueron libros, artículos de revistas científicas y tesis doctorales.

La información encontrada permitió realizar el estudio y análisis de contenido de los fundamentos teóricos de los precursores de la administración, de igual manera con los elementos relacionados con los distintos tipos de gestión que se deben poner en práctica en las empresas. Este estudio confirma la importancia de llevar a cabo una buena administración que permita la mejora continua de sus procesos, la optimización de sus recursos para alcanzar el desarrollo y éxito empresarial.

\section{RESULTADOS}

\section{Teorías administrativas}

La teoría administrativa conduce por una serie de bases conceptuales que guían pensamiento administrativo, pero cuando existen diversas teorías todas bien fundamentadas es importante definir una doctrina que permita la generación de nuevas ideas para aplicar en nuevos contextos.

Torres (2014) explica que la administración ha tenido aportes significativos de científicos y de diversas teorías, la cual refiere que la administración es el resultado histórico acumulado de la contribución de científicos en múltiples disciplinas. La filosofía nutre a todas las ciencias y estas, a su vez, facilitan la sistematización de un nuevo conocimiento que apareció a principios del siglo pasado con el nombre de administración. 
La evolución administrativa adquirió el nombre de teoría general de la administración, la que se divide en teorías específicas: administración financiera, administración de ventas y mercadotecnia (MKT), administración de operaciones y otras que facilitan los quehaceres de la administración pública, la administración de negocios y la administración de organizaciones no lucrativas (Torres Hernández, 2014).

El aporte de las ciencias es esencial para la construcción de nuevo conocimiento, las ciencias se necesitan unas a las otras, complementándose entre sí, cada una aporta al cumplimiento de un propósito. La Administración es interdisciplinaria, recibe el apoyo de todas las ciencias y las teorías, que nutren, que sirven como instrumento y como complemento para el logro de los objetivos de cada empresa u organización. La práctica administrativa va acompañada de las finanzas, la contabilidad, manejo de personal, entre otras disciplinas.

Es importante explicar los cambios en las prácticas administrativas, de acuerdo al estudio de la Escuela de Negocios de Harvard muestra en el tiempo algunos cambios, acompañado de sus acontecimientos según sea la práctica desde el año 1922 al 2000, según Torres (2014), detalla:

El nombre de la práctica ha cambiado, pero no así la práctica financiera que se mantuvo con el mismo nombre, estable, aunque con énfasis en distintos puntos. En 1922 la mira estaba puesta en las inversiones públicas, pues los compradores las veían como equitativas en sus ganancias, pero ya para el año 2000, la atención se puso en los productos derivados. (pág. 18)

El origen de la administración data de mucho tiempo atrás con un enfoque clásico y contemporáneo, Bateman y Snell (como se citó en Torres (2014)):

Desde el punto de vista de Thomas S. Bateman y Scott A. Snell, estos autores sitúan lo relevante del origen de la administración en el año 1100 a.C., por lo que sería administración empírica y es a partir de finales del siglo XIX que daría inicio la administración sistematizada. (pág. 19)

Los enfoques teóricos que sientan las bases para la gestión administrativa están: la teoría científica de la administración, teoría clásica de la administración, teoría de las relaciones humanas, teoría científica del comportamiento organizacional, teoría de sistemas, teoría de la burocracia y la teoría de la contingencia.

Koontz, Weihrich y Cannice (2012) explican que en la evolución del pensamiento administrativo "se cuenta con significantes contribuciones y practicantes de la administración. En la administración científica destacan Frederick Taylor; la teoría de la administración operacional moderna, de Henri Fayol; y los estudios Hawthorne, de Elton Mayo y F.J. Roethlisberger" (pág. 16). 


\section{La teoría científica de la administración}

Araujo (2015) señala que:

La administración científica recibe ese nombre al implementar el método científico en lugar de seguir con la práctica del ensayo y error como se había venido haciendo desde la antigüedad (Jiménez, 1995), o como la denominaba el propio Taylor (1983) la administración de iniciativa y estímulo. (pág. 168)

Chiavenato (2007) la administración científica de Taylor explica lo siguiente: La administración científica es una combinación de "ciencia en lugar de empirismo; armonía en vez de discordia; cooperación en vez de individualismo; rendimiento máximo en lugar de producción reducida. En fin, desarrollo de cada hombre para alcanzar mayor eficiencia y prosperidad" (pág. 49).

El principal aporte de Taylor consiste en la propuesta científica que permite elevar la productividad para alcanzar la eficiencia productiva, con una mejor remuneración a los trabajadores con la utilización del método científico. Entre los principios destacan la aplicación de la ciencia, creación de la armonía, cooperación de grupo, el logro de producción optima y el desarrollo de trabajadores (Koontz, Weihrich, \& Cannice, 2012).

Los aportes de Taylor fueron muy significativos para la administración, los cuales siguen vigentes en la práctica empresarial. Menciona que toda empresa $\mathrm{u}$ organismo necesita de personal apto en el desempeño de sus actividades, realizadas en coordinación con equipos para el logro de los objetivos en busca de la eficiencia. El éxito está en seleccionar al candidato adecuado desde el proceso de reclutamiento, la selección, hasta la contratación.

\section{Teoría clásica de la administración}

La teoría clásica hace énfasis en la estructura y en las funciones que debe tener una organización para alcanzar la eficiencia, esta se inicia con Fayol en el año 1916, en Francia.

La Doctrina Administrativa tiene como principal generador a Henry Fayol quien construye el paradigma del proceso administrativo y las áreas funcionales. Martínez (2005) afirma que: "La función básica de la Administración, como práctica, es la de seleccionar objetivos apropiados, guiar y dirigir a la organización hacia su logro" (pág. 70).

La contribución de Fayol gira en relación a la división de las actividades industriales en seis grupos: técnico, comercial, financiero, de seguridad, contable y gerencial. Reconoció la necesidad de enseñar administración. Formuló 14 principios de la administración como autoridad y responsabilidad, unidad de mando, cadena de escala y esprit de corps. Fayol es conocido como el padre de la teoría de la administración moderna (Koontz, Weihrich, \& Cannice, 2012). 
Los aportes de Fayol se implementan hoy en día en las empresas, facilitando la ejecución de las funciones administrativas como son la planeación, organización, integración, dirección y el control, a través de la división del trabajo. Es importante destacar, que la Administración es aplicada en cualquier ámbito y a cualquier tipo de empresa se lucrativa y no lucrativa, por su característica principal como es la universalidad.

\section{Teoría de las Relaciones Humanas}

Chiavenato (2007) refiere que la Teoría de las Relaciones Humanas (o Escuela Humanista de Administración) "surgió en Estados Unidos, como consecuencia de las conclusiones del experimento de Hawthorne, y fue desarrollada por Elton Mayo y colaboradores. Fue un movimiento de reacción y oposición a la Teoría Clásica de la Administración" (pág. 87).

La principal contribución a la administración fue estudios famosos en la planta Hawthorne de la Western Electric Company sobre la influencia de las actitudes y relaciones sociales de los grupos de trabajo en el desempeño.

Es importante destacar que los aportes de esta teoría en la actualidad, ya que, vienen a fortalecer el trabajo administrativo, en busca de la eficiencia productiva de la empresa, a armonizar el ambiente laboral entre los trabajadores y los jefes, las condiciones dignas y adecuadas, el clima de confianza y seguridad entre los miembros, elementos importantes e indispensables para el desempeño laboral de cualquier individuo.

\section{Teoría científica del comportamiento organizacional}

La teoría del comportamiento tiene su mayor exponente con "Herbert Alexander Simón. En el desarrollo de esta teoría cuenta con los autores Chester Barnard, Douglas McCgregor, Rensis Likertt y Chris Argyris" (Jaramillo Rivas, pág. 2).

Robbins y Judge (2009) expresan que el comportamiento organizacional también es conocido por la abreviatura $\mathrm{CO}$, el cual es definido como un campo de estudio que investiga el efecto de los individuos, grupos y estructura tienen sobre el comportamiento dentro de las organizaciones, cuyo propósito es de aplicar el conocimiento para la mejor y alcanzar la efectividad de las organizaciones.

Es fundamental que los directivos y jefes de áreas conozcan el comportamiento de sus colaboradores en los tres niveles, ya que les facilita en predicción, que harán los trabajadores a lo interno de la empresa, conocer la conducta de los individuos y grupos, y la comprensión de las relaciones interpersonales.

En las empresas al emplear el CO les permitirá identificar las habilidades de los colaboradores a nivel individual y grupal, pero no solo se trata de 
observar y comprender, sino también que permita a los directivos tomar decisiones para ofrecer un ambiente de armonía y este lleve a la eficiencia de los colaboradores, es por ello, que en toda empresa ya sea micro, pequeña, mediana aplique esta teoría.

\section{Teoría de los sistemas}

En cuando al surgimiento de la teoría de sistemas Chiavenato (2007) indica lo siguiente:

La TGS (Teoría de sistemas) surgió con los trabajos del biólogo alemán Ludwíg von Bertalanffy. La TGS no busca solucionar problemas o intentar soluciones prácticas, sino producir teorías y formulaciones conceptuales para aplicaciones en la realidad empírica. TGS afirma que se debe estudiar a los sistemas globalmente, involucrando a todas las interdependencias de sus partes. El agua es diferente del hidrógeno y del oxígeno que la constituyen. (pág. 410)

Los aportes de la teoría de sistemas a las empresas $u$ organizaciones son muy significativos y relevantes, ya que ha brindado herramientas para la solución de problemas en los diferentes niveles de las empresas. Las micro, pequeñas, medianas y grandes empresas son consideradas como un sistema donde cada una de sus partes interactúa entre sí, es decir cada una de las áreas tiene relación directa con las otras áreas, todas trabajan en armonía con propósitos para alcanzar una serie de objetivos, todo esto es visto y considerado como un todo en conjunto con los colaboradores que operan de los diversos sistemas administrativos.

\section{La teoría de la burocracia}

El principal promotor de la teoría burocrática fue Max Weber, quien definió el término como la forma más eficiente de la organización, tomando en cuenta al sistema estatal y sus dependencias, pensando en los cambios de la sociedad y las empresas. Según Petrella (2007), Max Weber visualizó a las empresas como organismos eficientes por excelencia, donde se resuelven las problemáticas sociales y empresariales de forma racional y eficiente, diseñada para desempeñarse con eficiencia para el logro de los objetivos por los cuales fue creada.

Petrella (2007) refiere a Max Weber, quien explica que la burocracia se basa en la formalización (tanto de las normas como de los procedimientos) y la centralización (definición de la jerarquía y la autoridad), ambas interpretadas desde "la racionalidad formal", origen del "orden legítimo" que han de establecer las normas jurídicas.

De igual manera establece que la legitimidad puede ser "carismática" (se basa en la santidad, heroicidad o en otras cualidades que concurren de forma especial en una determinada persona), "tradicional" (deriva del carácter 
sagrado que caracteriza a determinadas tradiciones, usos o costumbres) y "racional" (que encuentra su fundamento en el principio de legalidad propio del ordenamiento jurídico). El ejemplo máximo de racionalidad lo constituye la "organización burocrática".

Es notable el aporte de la teoría burocrática a la administración, en la actualidad en toda empresa e institución es fundamental la ubicación jerárquica del elemento humano con habilidades, destrezas y con conocimiento y dominio en su campo profesional, quienes se desempeñan para el logro de los objetivos y metas de la empresa, resolviendo los conflictos de forma racional.

\section{La teoría de contingencia}

La teoría de la contingencia es la última en el proceso de evolución de las teorías administrativas, la cual se vio enriquecida por todas las anteriores, de igual manera tuvo otros aportes por los hallazgos en de diversas investigaciones que mostraron la estrecha dependencia de la organización frente a los acontecimientos y sus probabilidades de ocurrencia. Uno de los pioneros fue Joan Woodward (Sociólogo industrial), quien plantea los siguientes hallazgos:

Realizó en la serie: Managementand technology, el hallazgo en el cual se afirma que las estructuras de éxito eran las más típicas de su clase de sistema técnico, es decir, aquellas cuyas medidas de parámetros de diseño se alejaban menos de la media. Fue ella quien introdujo la noción de la teoría de la contingencia, según la cual: la efectividad de la organización surge a raíz de una correspondencia entre la situación y la estructura. (Gutiérrez, Soto, Castillo, \& Pinto, 2003, pág. 71)

Otro aporte a esta teoría la dan Paul Lawrence y Jay Lorsch (como se citó en Gutiérrez et al., (2003)), quienes explican que en las diferencias estructurales, no existe una única estructura idónea, sino muchas distintas que cambiaban según sean las condiciones de la organización. Por estos hallazgos los autores formularon la Teoría de Contingencia señalando que: "no existe una manera de organizar única y mejor; por el contrario, las organizaciones necesitan ser sistemáticamente adecuadas a las condiciones ambientales" (pág. 71).

Los aportes de la teoría de la contingencia se orientan a la estructura de las empresas, estas se ven condicionadas por el ambiente que la rodea, es decir los elementos externos a esta y a un nivel interno por la implementación del proceso administrativo.

En la actualidad un elemento influyente en las empresas es la tecnología, la que permite mejorar los procesos de producción y también es utilizada para la comercialización, determinando así su nivel de desempeño y clasifica a las empresas por categorías, en el aspecto tecnológico. 
En cuanto a la implementación del proceso administrativo, este permite determinar el nivel de desempeño de las empresas y otorgar una clasificación de eficiencia o ineficiencia en sus procesos.

Las empresas se encuentran en constante cambio, están en constante actualización, revisión y mejora continua de sus procesos, los que van a incidir en la estructura y facilitaran el logro de los objetivos a un corto, mediano o largo plazo.

\section{La gestión empresarial}

Los aportes que han brindado los diferentes enfoques o teorías expuestas anteriormente han contribuido a que las micro, pequeñas, medianas y grandes empresas cuenten con diversas herramientas y estrategias para aplicarlas en la gestión empresarial, estas contribuyen al manejo eficiente de los recursos, al crecimiento y desarrollo y la sostenibilidad en el sector que están establecidas.

A través de la adecuada implementación de la gestión empresarial se logran los objetivos de la empresa, por ello, es indispensable que los propietarios estén conscientes de la importancia que tiene la gestión administrativa: "Un elemento clave de la gestión empresarial, es la administración. Su importancia radica en atender las necesidades internas y externas de la empresa, por lo cual es necesario planear, organizar, dirigir y controlar, a fin de garantizar el éxito organizacional" (Córdoba Gómez, Vanegas Michel, Orozco Raymundo, \& Palomares Rodríguez, 2018, pág. 760).

Villasmil, Castro y Torres (2010), Hernández, Marulanda y López (2014), Tabares y López (2015) refieren los tipos de gestión empresarial que toda empresa debe aplicar: gestión administrativa, gestión financiera contable, gestión de comercialización, gestión de abastecimiento, gestión del talento humano, gestión del conocimiento e innovación y la gestión empresarial como sistema.

\section{Gestión administrativa}

Es evidente que para dirigir una empresa se deben de seguir una seria de pasos o etapas, así lo plantea los autores Koontz y Weihrich Mendoza (como se citó en Mendoza et al., (2020)) "El proceso administrativo comprende las actividades interrelacionadas de: planificación, organización, dirección y control de todas las actividades que implican relaciones humanas y tiempo" (Mendoza Alcívar, Álvarez Gómez, \& Rivera Segura, 2020, pág. 3).

Villasmil, Castro y Torres (2010) "La gestión administrativa se relaciona con el manejo cotidiano de los recursos en el marco de una estructura que distribuye atribuciones y responsabilidades que define el esquema de división del trabajo" (pág. 6). 
La implementación adecuada del proceso de la gestión administrativa contribuye al manejo y el funcionamiento de todas las operaciones de cualquier empresa sin importar su tamaño o giro del negocio. Es un proceso integral que inicia con la planeación y finaliza con el control, es sistemático y necesario para analizar la situación actual de la empresa en los diferentes niveles, también, para establecer nuevas metas y realizar cambios en los procesos, para la organización y la conducción del personal que está a cargo de las actividades e indispensable para tomar decisiones.

Lo anterior descrito, enfatiza la importancia que las micro, pequeña, medina y grandes empresas lleven a cabo la gestión administrativa, ya que, les permitirá planificar, organizar, integrar al personal, dirigir y controlar las actividades hacia el logro de los objetivos en un corto, mediano y largo plazo.

La sostenibilidad de las mipymes depende mucho de la gestión administrativa, de sus recursos internos, al respecto Molina (2019) plantea que: "En ese sentido los aspectos internos tienen mayor relevancia para la supervivencia de las micro y pequeñas empresas porqué debido a su tamaño la participación del empresario y colabores es ineludible (pág. 294).

\section{Gestión financiera contable}

Los registros contables permiten disponer de información real para la toma de decisiones en tiempo real, también para conocer la disponibilidad de los recursos con los que cuenta la empresa y la situación actual en cuanto a pérdidas o ganancias, por ello es indispensable realizar esta actividad en las empresas.

Los administradores hábiles deben saber extraer de la información contable para tomar decisiones en cuanto al comportamiento del capital en relación con las utilidades o pérdidas obtenidas durante el ejercicio, la adecuada inversión en bienes propios, conocer el monto de los ingresos y egresos, conocer a cuánto ascienden las deudas y sus plazos, etc., (Guerrero Reyes \& Galindo Alvarado, 2014). Toda esta información enriquecerá los conocimientos personales, así como aquellos que le servirán para rendir información a los dueños de las empresas.

Las empresas deben de realizar los registros contables, ya que estos son esenciales para el éxito del negocio, estos ayudan a tomar decisiones, a evaluar de forma permanente las finanzas y garantizar la rentabilidad. Sin una correcta contabilidad ninguna empresa sabrá cuánto dinero entra o sale, si hay pérdidas o ganancias, continuar con el flujo de las operaciones y no podrán planear el crecimiento futuro.

La empresa que aplique la contabilidad financiera conocerá el comportamiento de los costos en la elaboración de un producto y la oferta de un servicio, 
determinará con precisión el precio de venta de estos, facilitando así el cálculo de los movimientos contables y de los estados financieros en tiempo real.

\section{Gestión del talento humano}

En la actualidad el elemento humano trasciende en importancia dentro de la empresa, este es el encargado de realizar diversas actividades y con ellas el logro de los objetivos de la misma. Para que los colaboradores alcancen los objetivos propuestos por los directivos deben de ser dirigidos o gestionados adecuadamente, siguiendo un proceso desde la selección hasta el seguimiento de su trabajo.

Chiavenato (como se citó en Galarza et al., (2020)) plantea un modelo de gestión administrativa con enfoque en el talento humano de las organizaciones. Mencionan que el comportamiento de las personas a lo interno de las empresas influye positiva o negativamente, y por ello resulta importante la existencia de una adecuada gestión del talento humano, y que esta se ejecute prudentemente, con profesionalismo en procesos básicos como el de selección, evaluación, capacitación y monitoreo.

En la Gestión del talento humano las teorías concernientes al control de gestión de la organización han ido evolucionando a medida que se han descubierto y considerado nuevas variables que afectan las actividades de la organización y de sus miembros, tanto a nivel interno como externo. Estas también se han transformado de acuerdo a la propia realidad de las organizaciones. El desarrollo ha estado unido a los avances de la literatura especializada, así como a las transformaciones vividas por la sociedad, a los cambios en los mercados, a su internacionalización y al incremento de la competitividad (Pérez Mayo, Vasquez García, \& Levin Kosberg, 2015).

La gestión del talento humano permite a las micro, pequeña, mediana y grandes empresas generar un ambiente laboral adecuado, el que propicia la productividad, el compromiso y la motivación del personal, también, permite identificar las necesidades que tienen los colaboradores en cada área de trabajo para orientar las metas y los objetivos de la empresa.

De igual manera, se capacita al personal, este mecanismo permite el desarrollo continuo de los colaboradores, favorece a la ejecución de programas de bienestar, resolver conflictos que surjan, así como el fortalecimiento de las relaciones interpersonales. De aplicarse una adecuada gestión del talento humano facilita la mejora continua de los procesos y por consiguiente obtener avances en la competitividad en el sector que atienden las empresas. 


\section{Gestión de abastecimiento}

La gestión de abastecimiento o la logística de aprovisionamiento juega un papel fundamental en el buen funcionamiento de una empresa. Su objetivo es controlar los suministros a fin de satisfacer las necesidades de los procesos operativos.

La gestión de la cadena de suministro radica en sincronización y alineación de los procesos logísticos y sus actores (proveedores, productores, distribuidores y clientes), con el propósito de satisfacer las necesidades de los clientes y generar un valor agregado al producto, haciendo un uso eficiente de los recursos (Gómez Montoya, Zuluaga Mazo, Ceballos Atehourtua, \& Palacio Jiménez, 2019).

La aplicación de una buena gestión de la cadena de suministros se ve reflejada en la rentabilidad de la empresa, la productividad y la satisfacción del cliente. Esta gestión requiere de una planeación en conjunto, para satisfacer las necesidades del mercado, partiendo siempre de las necesidades del cliente y orientando los procesos para lograr satisfacerlas, Heikkilä (como se citó en Gómez, Zuluaga, Ceballos (2019)).

Debido a la fuerte competencia en el ámbito empresarial la gestión de abastecimiento ha cobrado mucha importancia en la actualidad, ya que juega un papel esencial en la eficiencia de las operaciones, los propietarios o directivos de las empresas buscan a personal con habilidades y conocimientos en el área para obtener el movimiento de bienes desde el suministro de materia prima, la venta y la entrega del producto en manos del consumidor final. Entre de los objetivos que obtienen las empresas al implementar este tipo de gestión es la eficacia, a menor costo, sin afectar la calidad y brindando un buen servicio al cliente.

\section{Gestión del conocimiento e innovación}

Hernández, Marulanda y López (2014), consideran la necesidad de establecer un sistema formal de gestión del conocimiento que conduzca a la innovación mediante la utilización de diversas herramientas:

Es necesario que las empresas incorporen la gestión del conocimiento para innovar mediante el manejo direccionado de procesos de conocimiento (identificar, compartir, generar, retener y aplicar conocimiento), formando al capital humano en gestión de la información y la documentación, en gestión de la innovación y el cambio, en gestión del aprendizaje organizacional, en diseño de herramientas digitales y en gestión de la comunicación.

Se deben formular acciones para consolidar el uso de las TIC en función de gestión de información y conocimiento, la apropiación de herramientas de trabajo colaborativo, de gestión de proyectos, de gestión de relaciones sociales y de modelamiento y simulación. (pág. 4) 
Escorsa Castells et al., (2003) especifican:

La innovación supone, pues, que el directivo introduzca o permita voluntariamente un poco de desorden y de imprevisto en el funcionamiento de su empresa, incorporando un nuevo nivel, una nueva dimensión: la de la mutación, es decir, la capacidad de crear y de cambiar. Los directivos que triunfan quieren conseguir cosas nuevas, y se apasionan por el cambio. (pág. 117)

La globalización ha generado cambios significativos en las actividades de la empresa, generando un ambiente competitivo y exigente para las empresas, es por ello que la gestión del conocimiento y la innovación juegan un papel fundamental en este proceso de adaptación y cambio en el entorno empresarial. La gestión del conocimiento y la innovación es indispensable en las empresas, ya que, son recursos para el intercambio de conocimiento en conjunto con la experiencia de todos los miembros, a través de diversas estrategias de aprendizaje para mantener en constante actualización al personal y así obtener un impacto en la calidad, la productividad y por ende en el desarrollo de la empresa.

\section{Gestión de comercialización}

La gestión de comercialización se dirige para satisfacer necesidades del mercado que ha seleccionado atender. Este mercado son todos los clientes potenciales que tienen una necesidad o deseo en común. Toda empresa debe de establecer objetivos y estrategias para determinar que puede ofertar a sus clientes, elegir la forma en cómo llegarle al cliente para que a través de la compra de ese producto o servicio puedan satisfacer esas necesidades o deseos.

El proceso de gestión de comercialización en una empresa requiere la decisión en cuanto a la mezcla de mercadotecnia, la cual comprende cuatro elementos: Producto, precio, plaza y promoción.

Domínguez (2009), explica que el principal propósito de un producto es satisfacer una necesidad al conceptualizarlo lo define de la siguiente manera: "El término producto aplicado en el contexto de la receta de Marketing, abarca todo lo que la empresa ofrece a sus clientes con el fin de satisfacer sus necesidades" (pág. 41).

Para que una empresa logre una buena gestión comercial necesita realizar un estudio del mercado que pretende atender, plantearse objetivos, identificar los medios de distribución entre otros elementos. Se sigue un proceso con una serie de pasos para obtener una meta de ventas, con el objetivo de satisfacer a los clientes.

El proceso que se sigue parte de la segmentación de mercado, para Feijoo, Guerrero y García (2018) "La segmentación es el proceso de dividir el 
mercado total para un producto en particular o una categoría de productos en segmentos o grupos relativamente homogéneos" (pág. 16).

Una vez que se ha establecido el segmento del mercado, se debe de indicar la posición que ocupará en los segmentos. El posicionamiento no es crear algo diferente sino manipular la mente del cliente de acuerdo a lo que ya existe y reordenar los vínculos existentes.

Cuando ya se definió el mercado, se establecido el posicionamiento, se prosigue con la utilización de estrategias de mercadeo, en una empresa son de vital importancia para competir en el mercado, Tabares et al, (2015). La venta se puede realizar desde un mostrador, por el sistema de autoservicio o mediante la combinación de ambos sistemas, es visible el esfuerzo de la planeación y ejecución de las actividades que realizan los ejecutivos en venta.

El comerciante no solo debe de dedicarse a vender y comprar productos, este debe de emplear tácticas que atraigan y estimulen a los clientes a realizar la compra, de igual manera pueden contratar a un experto en Marketing para que ejecute actividades para el logro de los objetivos y aumentar el conocimiento que el público tiene de la empresa, el de sus productos y pueda proporcionar, variedad, interés y estímulos, que lleven a promocionar las ventas. (Tabares Hoyos \& López Cardona, 2015).

La mercadotecnia moderna requiere algo más que desarrollar un buen producto, fijarle un precio atractivo y ponerlo al alcance de sus clientes meta, las compañías también deben comunicarse con éstos y lo que dicen nunca debe dejarse al azar.

\section{La gestión empresarial como sistema}

Los sistemas están conformados por partes y elementos que conforman un todo, las empresas están estructuras por áreas y departamentos donde cada uno tiene diferentes objetivos, pero el fin es en común. Es importante que las unidades o áreas de las empresas estén en constante comunicación e intercambiando información para conocer la situación actual del negocio, así, se podrá tomar decisiones para encontrar mejores soluciones y realizar cambios pertinentes ajustados a las metas y objetivos indicados por la gerencia.

El control de gestión en una empresa no solo comprende medir los resultados obtenidos, sino que permite diagnosticar e identificar las oportunidades de mejora. Hernández Torres et al., (2006) refiere que para administrar transformaciones en la empresa, el control no se puede reducir únicamente a comprobar si los resultados se han alcanzado o no, sino que requiere también apoyarse en una práctica de diagnóstico permanente, tratando de identificar las posibilidades de mejora y buscando metas de desempeño cada vez más altas. 
El diagnóstico se incluye como una función de los Sistemas de Control de Gestión modernos (SCG), con el propósito de conocer por completo el funcionamiento de la organización y para comprender los mecanismos que explican el comportamiento de los procesos que condicionan los resultados. De esta manera se conciben los posibles cursos de acción para mejorar el desempeño, permitiendo establecer los relaciones que se vinculan con las variables técnicas, organizativas y sociales con el resultado económico de la empresa, todo esto en relación con el nuevo modelo empresarial orientado al aprendizaje (Hernández Torres \& Acevedo Suárez, 2006).

La conducción de la información de la empresa es transcendental para el éxito o el fracaso. Conocer la situación actual del negocio permitirá planificar, organizar, integra al personal, dirigir y controlar, cada uno de los procesos, actividades y estrategias que se establecen en cualquier empresa, indistintamente de su giro o mercado a que este dirigida.

\section{CONCLUSIONES}

En este artículo científico se desarrollaron las diferentes teorías que dieron origen y aportes significativos a la Administración, los precursores más relevantes que marcaron la historia administrativa, quienes permitieron que se fortaleciera como ciencia y demostraron su aplicabilidad en los distintos ámbitos de la industria para obtener mejores resultados organizacionales en el corto, mediano y largo plazo. De igual manera, se abordó los diferentes tipos de gestión empresarial que debe de aplicar cualquier tipo de empresa, para el cumplimiento de sus metas, objetivos, para alcanzar la eficiencia y eficacia de los procesos y los recursos, de igual manera para alcanzar el posicionamiento, desarrollo, éxito y sostenibilidad empresarial.

Se hace referencia a la importancia que conlleva a la implementación adecuada de las gestiones, ya que estas contribuyen al manejo y el funcionamiento de todas las operaciones de cualquier empresa sin importar su tamaño o giro del negocio, el análisis de la situación actual de la empresa en los diferentes niveles jerárquicos, para el establecimiento de las metas y cambios en procesos, conducción de personal y en la toma de decisiones. Lo anteriormente descrito enfatiza la importancia que las micro, pequeña, medina empresas implementen los tipos de gestiones empresariales, ya que, les permitirá planificar, organizar, integrar al personal, dirigir y controlar las actividades hacia el logro de los objetivos en un corto, mediano y largo plazo.

La práctica de una buena gestión empresarial por parte de los empresarios, facilita a la mejora en los procesos, en la productividad, al logro de los objetivos a través de los colaboradores, afrontar con preparación y 
conocimiento los retos que amenacen la estabilidad del negocio, de igual manera, posicionarse y ser competitivos en el sector que operan, asegurando el futuro y el éxito de la empresa.

\section{REFERENCIAS}

Araujo, A. J. (Enero de 2015). Cameralismo: un antecedente más en la evolución de la administración como ciencia. (V. Gerencial, Ed.) redalyc. org(1), 165-180. Recuperado el 07 de Septiembre de 2020, de https:// www.redalyc.org/articulo.oa?id $=465545898008$.

Chiavenato, I. (2007). Introducción a la teoría general de la administración Séptima edición (Séptima edición ed.). México, México: McGRAW-HiLL/ INTERAMERÍCANA EDITORES, S.A. DE C.V.

Córdoba Gómez, S., Vanegas Michel, L., Orozco Raymundo, A., \& Palomares Rodríguez, I. D. (2018). Análisis de las áreas de la Gestión Empresarial en MIPYMES. Academia Journals 2018, Vol. 10, No. 3, 2018, 759-762. Recuperado el 11 de Octubre de 2020, de http://search.ebscohost.com/ login.aspx? direct $=$ true $\& d b=$ fap\&AN $=144696746 \&$ lang $=$ es \&site $=$ eho st-live

Escorsa Castells, P., \& Valls Pasola, J. (2003). Tecnología e innovación en la empresa. Catalunya, Barcelona: EDICIONS UPC. Obtenido de https:// elibro.net/es/ereader/unanmanagua/61428? page $=117$

Galarza Villalba. , M. F., Mora Romero, J. L., \& Zambrano Campi, D. (2020). Gestión administrativa, la sostenibilidad de las agrupaciones rurales en la provincia de los Ríos - Ecuador. Revista Dilemas Contemporáneos: Educación, Política y Valores., 21. Recuperado el 26 de Septiembre de 2020, de http://search.ebscohost.com/login.aspx?direct=true\&db=fap\& $\mathrm{AN}=141887637$ \&lang $=$ es\&site $=$ ehost-live

Gómez Montoya, R. A., Zuluaga Mazo, A., Ceballos Atehourtua, N., \& Palacio Jiménez, D. (2019). Gestión de la cadena de suministros y productividad en la literatura científica. I+D Revista de Investigaciones, 34. Recuperado el 12 de Octubre de 2020, de https://www.researchgate. net/publication/333540042_Gestion_de_la_cadena_de_suministros_y_ productividad_en_la_literatura_cientifica

Guerrero Reyes, J. C., \& Galindo Alvarado, J. F. (2014). Contabilidad para administradores. México: Grupo editorial Patria. Obtenido de https:// elibro.net/es/ereader/unanmanagua/39381? page $=17$

Guerrero, J., García, J., \& Feijoo, I. (2018). Marketing aplicado en el sector empresarial. Machala - Ecuador: UTMACH, 2018. Obtenido de Marketing aplicado en el sector empresarial: http://repositorio.utmachala.edu.ec/ bitstream/48000/14270/1/Cap.2-Segmentaci\%C3\%B3n\%20del\%20 mercado.pdf

Gutiérrez, A., Soto, N. Y., Castillo, L. J., \& Pinto, J. D. (2003). Ajuste, estructura y ambiente como factores claves en la teoría de contingencias. scielo 
Estudios gerenciales, 88, 71. Recuperado el 10 de Octubre de 2020, de http://www.scielo.org.co/pdf/eg/v19n88/v19n88a04.pdf

Hernández Torres, M., \& Acevedo Suárez, J. (2006). Un modelo de diagnóstico para el control de gestión empresarial. 8. Recuperado el 25 de Septiembre de 2020, de http://search.ebscohost.com/login.aspx? direct=true\&db=a sn\&AN $=25591175 \&$ lang $=$ es\&site $=$ ehost-live

Hernández, A., Marulanda, C., \& López, M. (2014). Análisis de capacidades de gestión del conocimiento para la competitividad de Pymes en Colombia. Información tecnológica, 25(2). Recuperado el 08 de Octubre de 2020, de https://scielo.conicyt.cl/scielo.php?pid=S0718$07642014000200013 \&$ script $=$ sci_arttext

Jaramillo Rivas, M. (Sin fecha). Teoría del comportamiento. (U. N. CARIBE, Ed.) Recuperado el 10 de Octubre de 2020, de http://umc.edu.ve: http:// umc.edu.ve/miaramillo/Unidades/Unidad\%20VI/Guia/Guia.pdf

Koontz, H., Weihrich, H., \& Cannice, M. (2012). Administración Una perspectiva global y empresarial. México: Mc Graw Hill Educación.

Martínez Crespo, J. (Julio de 2005). Administracion y Organizaciones Su desarrollo evolutivo y las propuestas para el nuevo siglo. (https://revistas. udem.edu.co/, Ed.) Semestre Económico, 8(16), 67-97. Recuperado el 07 de Septiembre de 2020, de https://revistas.udem.edu.co/index.php/ economico/article/view/1099

Mendoza Alcívar, M. A., Álvarez Gómez, L. K., \& Rivera Segura, G. N. (2020). Los procesos administrativos y su contribución en el fortalecimiento de las MiPymes del cantón Quevedo. Revista Dilemas Contemporáneos: Educación, Política y Valores, 13. Recuperado el 26 de Septiembre de 2020, de http://search.ebscohost.com/login.aspx?direct=true\&db=fap\& $\mathrm{AN}=141887633$ \&lang $=$ es\&site $=$ ehost-live

Molina Corral, L. A., Piñón Howlet, L. C., Sapién Aguilar, A. L., \& Gallegos Cereceres, V. M. (2019). Análisis de las Habilidades Administrativas y de Gestión en las Micro y Pequeñas Empresas de la ciudad de Chihuahua. Nova Scientia, 11(22), 293-322. Recuperado el 25 de Septiembre de 2020, de http://search.ebscohost.com/login.aspx? direct=true\&db=asn\& $\mathrm{AN}=137197180$ \&lang $=$ es\&site $=$ ehost-live

Pérez Mayo, A. R., Vasquez García, A. W., \& Levin Kosberg, S. (Julio de 2015). El control de gestión y el talento humano: conceptos y enfoques. Universidad \& Empresa, 17(29), 13-33. Recuperado el 12 de Septiembre de 2020, de https://www.redalyc.org/articulo.oa? id=187243745002

Petrella, C. (2007). Análisis de la teoría burocrática, Aportes para la comprensión del modelo burocrático. Recuperado el 10 de Octubre de 2020, de www.fing.edu.uy: http://biblioteca.esucomex.cl/RCA/ An\%C3\%A1 lisis\%20de\%20la\%20teor\%C3\%ADa\%20burocr\%C3\%A 1 tica. pdf

Robbins, S. P., \& Judge, T. A. (2009). Comportamiento organizacional (Vol. DECIMOTERCERA EDICIÓN ). México: Pearson Educación de México S.A de C.V. Recuperado el 10 de Octubre de 2020, de https://elibro.net/es/ ereader/unanmanagua/39534?page $=44$

Rubio Domínguez, P. (2009). ¿Cómo llegar a ser un experto en Marketing? (I. 


\section{E. EMPRESARIAL, Ed.) Madrid, España.}

Tabares Hoyos, R., \& López Cardona, D. (Diciembre de 2015). Estrategias comerciales para la competitividad en una ciudad intermedia: caso Manizales. https://revistageon.unillanos.edu.co/, 2(2), 85. Recuperado el 19 de Septiembre de 2020, de https://revistageon.unillanos.edu.co/ article/view/99

Torres Hernández, Z. (2014). Teoría generalde laAdministración. México: GRUPO EDITORIAL PATRIA. Obtenido de https://editorialpatria.com.mx/ pdffiles/9786074386196.pdf

Villasmil, E., Castro, E., \& Torres, E. (Marzo de 2010). Gestión administrativa y los recursos financieros asignados para apoyo de la investigación. REDHECS: Revista electrónica de Humanidades, Educación y Comunicación Social(Año 5, №. 8, 2010), 4-17. Recuperado el 12 de Septiembre de 2020, de https://dialnet.unirioja.es/servlet/articulo? codigo $=3167932$ 